\title{
Transparency of Health Informatics Processes as the Condition of Healthcare Professionals' and Patients' Trust and Adoption: the Rise of Ethical Requirements
}

\author{
Brigitte Séroussi ${ }^{1,2}$, Kate Fultz Hollis ${ }^{3}$, Lina F. Soualmia ${ }^{4}$ \\ 1 Sorbonne Université, Université Sorbonne Paris Nord, INSERM UMR S1 142, LIMICS, Paris, France \\ 2 Assistance Publique - Hôpitaux de Paris, Hôpital Tenon, Paris, France \\ 3 Oregon Health \& Science University Department of Biomedical Informatics and Clinical \\ Epidemiology, Portland, Oregon, USA \\ ${ }^{4}$ Normandie Université, Univ Rouen, LITIS EA 4108, Rouen, France
}

\begin{abstract}
Summary
Objectives: To provide an introduction to the 2020 International Medical Informatics Association (IMIA) Yearbook by the editors.

Methods: This editorial provides an introduction and overview to the 2020 IMIA Yearbook which special topic is: "Ethics in Health Informatics". The keynote paper, the survey paper of the Special Topic section, and the paper about Donald Lindberg's ethical scientific openness in the History of Medical Informatics chapter of the Yearbook are discussed. Changes in the Yearbook Editorial Committee are also described.

Results: Inspired by medical ethics, ethics in health informatics progresses with the advances in biomedical informatics. With the wide use of EHRs, the enlargement of the care team perimeter,
\end{abstract}

the need for data sharing for care continuity, the reuse of data for the sake of research, and the implementation of Al-powered decision support tools, new ethics requirements are necessary to address issues such as threats on privacy, confidentiality breaches, poor security practices, lack of patient information, tension on data sharing and reuse policies, need for more transparency on apps effectiveness, biased algorithms with discriminatory outcomes, guarantee on trustworthy Al, concerns on the re-identification of de-identified data.

Conclusions: Despite privacy rules rooted in the Health Insurance Portability and Accountability Act of 1996 (HIPAA) in the USA and even more restrictive new regulations such as the EU General Data Protection Regulation published in May 2018, some people do not believe their data will be kept confidential and may not share sensitive information with a provider, which may also induce unethical situations. Transparency on healthcare data processes is a condition of healthcare professionals' and patients trust and their adoption of digital tools.

\section{Keywords}

Informatics, medical; Health Information Technology; IMIA Yearbook of Medical Informatics; Artificial intelligence; Data sharing; Ethics

Yearb Med Inform 2020:7-10

http://dx.doi.org/10.1055/s-0040-1702029

\section{From Data Privacy Concerns to Trustworthy Artificial Intelligence}

Every year, the International Medical Informatics Association (IMIA) Yearbook choses a special topic to focus on a specific thematic area related to health informatics practices. After "Learning from experience: secondary use of patient data" in 2017, "Between access and privacy: challenges in sharing health data" in 2018, and "Artificial intelligence in health: new opportunities, challenges, and practical implications" in 2019, the special topic of the 2020 Yearbook appeared as a fitting culmination of the preceding topics. Embedding data access versus privacy concerns - especially in the case of data secondary use - on the one side and the new challenges of Artificial Intelligence (AI) in health applications, on the other side, the 2020 Yearbook special topic "Ethics in health informatics" (EHI) was obvious to the Yearbook Editorial Committee and supported by IMIA working group chairs and co-chairs.

Ethics may be defined as the moral principles that govern a person's behavior or the conducting of an activity. According to the Merriam Webster, ethics and morals are often regarded as synonyms. More specifically, medical ethics, which spirit is included in the
Hippocratic Oath, is known to rely on four pillars: (i) autonomy, e.g., patients but also physicians should keep their autonomy of thought, intention, and action when making decisions regarding health care procedures; (ii) justice, e.g., burdens and benefits of health care procedures, especially treatments, must be distributed equally to be fair with all players involved; (iii) beneficence, e.g., health care procedures are provided with the intent of doing good for the patient involved; and (iv) non-maleficence, e.g., health care procedures should not harm the patient involved.

Inspired by medical ethics, ethics in health informatics progresses with the advances in biomedical informatics. Previ- 
ously, EHI was essentially concerning data privacy. When there was one care provider for one patient, maintaining the security and confidentiality of patient records was essentially based on the conversational discretion of the health care provider and the physical security of paper-based medical records. The advent of electronic health records (EHRs) has raised new concerns about privacy. Now that a patient is being cared for by multiple care providers in different settings, data privacy concerns have been extended to include security and confidentiality issues at the moment of data sharing and exchange among the members of the healthcare team in charge of a given patient. Going further, the implementation of the Learning Health System (LHS) paradigm (an evidence-building system able to learn from every patient encounter "best practices seamlessly embedded in the delivery process and new knowledge captured as an integral by-product of the delivery experience" [1]) is another example of health information and data re-use that could, as in the rapid deployment of cloud computing, potentially lead to data privacy breaches.

Despite privacy rules rooted in the Health Insurance Portability and Accountability Act of 1996 (HIPAA) in the USA that restrict the sharing of identifiable health data, and even more restrictive new regulations such as the EU General Data Protection Regulation published in May 2018 (GDPR) that led to a renewed focus on balancing privacy and sharing of personal data, novel technologies have the potential to enable the automated collection and analysis of health data. Are patients aware about how their data are being used and by whom? For instance, patients and their family members are often not informed about or asked to consent to the use of AI-powered decision support tools - many of them unproven - in their care [2]. Is consent for personal healthcare data processing actually always collected? Is data processing performed the right way (data minimization, accuracy, storage limitation, etc.)? One can wonder whether healthcare data can be private when wearables and mobile apps collect health data that can be shared for advertising purposes in de-iden- tified form (often without any disclosure to, or consent from, the individuals using the applications) while it has been proven that re-identification is often possible with really low effort [3]. Anonymization is indeed difficult in regard to large data collections as (for example genetic) data may affect not only the privacy rights of individuals but also the rights of whole groups for ethnic or geographic reasons [4]. Furthermore, "Big Data" approaches challenge the established science paradigm, leading to new forms of empiricism that declare the creation of a science based on data rather than knowledge [5].

The European Commission has recently questioned the principle of a trustworthy Artificial Intelligence with the publication of Ethics Guidelines [6] putting forward a set of key requirements that AI systems should meet in order to be deemed trustworthy. Interestingly, one is about the need for human agency and oversight (human-in-the-loop, human-on-the-loop, and human-in-command) and the need for transparency (AI systems and their decisions should be explained in a manner adapted to the stakeholder concerned). Explainable AI has thus to progress if human healthcare providers have to explain to their patients how machine learning models often thought of as "black boxes" are reasoning behind their predictions to comply with the autonomy pillar of medical ethics. Another issue relies on the identification of ways in which a deep learning algorithm embeds racial, ethnic, gender, or other biases which shape or corrupt its results and erode public confidence in the implementation of AI systems. Lastly, there are also growing concerns about the "de-skilling" of physicians that could occur when some or all of the tasks become automated, such as a drop in a clinician's diagnostic accuracy.

Threats on privacy, confidentiality breaches, poor security practices, lack of patient information, tension on data sharing and reuse policies, need for more transparency on apps effectiveness, biased algorithms with discriminatory outcomes, guarantee on trustworthy AI, concerns on the re-identification of de-identified data, all these issues are critical to medical practice as some people may not seek care or share sensitive information with a provider if they do not believe their data will be kept confidential [7]. Studies have reported that patients may not disclose clinical information to healthcare providers to protect against the perceived EHR privacy and security risks despite EHR advantages for promoting quality of care [8]. On the other hand, insufficient use of digital technology in patient care, for research, or to support the development of a data-driven care system management may also induce, on a large scale, unethical situations. Indeed, not receiving benefit from AI applications that hold the promise of improving safety, fairness, and welfare is unethical. Besides, AI applications have the potential to reduce present-day discrimination caused by human subjectivity. For all these reasons, the special topic, Ethics in Health Informatics, for the 2020 IMIA Yearbook could not have been more timely.

\section{Highlights in this Year's Yearbook}

This year's keynote paper written by Kenneth W. Goodman presents an optimistic view of how the insights and analyses provided by ethics are currently incorporated by academic and health care institutions. This position is illustrated by different contemporary challenges including Artificial Intelligence and Machine Learning; Big Data, Data sharing and privacy; Duties to use and manage new technology; and Ethics and public policy. The keynote paper proposes commandments in the development and use of machine learning programs, including "Quality and standards are ethical issues", "Prevent and eliminate bias", "Use machine learning software for good and not evil", "Insist on and provide robust education and evaluation". Interestingly, the duties to use information technology because "its advantages outweigh its disadvantages" are illustrated by the Learning Health System paradigm that advocates the overarching duty to use tools that improve health. 
In keeping with the theme of this year's edition of the Yearbook, Dr Galvin and Dr DeMuro offer a survey in the Special Section about the issues regarding privacy protections and data ownership in mobile health (mHealth) technologies. They observe that the security of mHealth data storage and transmission remains a concern. Consumers are often uneducated regarding the ways a service may collect and transmit their data to third parties. They introduce the concept of a "health care fiduciary" as a means to protect the basic human right of privacy in an equitable fashion across a dynamic ecosystem.

Ethics in health informatics, as perceived though the lens of clinical research informatics (CRI), is described by Dr Anthony Solomonides in the survey paper of the CRI section. The paper proposes a personal view on Artificial Intelligence, Machine Learning, and Big Data Analytics and describes the shift of AI from logic to data with the counterpart of biases and currently unsatisfied though essential needs for explanation. The role of common data models is emphasized as a way to organize and store data in a highly standardized form, to foster clinical research on "real world data" and accelerating observational studies. Then, the section on phenotyping and cohort discovery introduces the issue of de-identification which efficacy is counterbalanced by the availability of data sets and methods allowing to link individuals in one data set to those in the supposedly de-identified collection leading to re-identification. Although blockchain has been suggested as a possible answer to the challenges of anonymous data sharing, it has not yet had wide adoption in the field.

In the History of Medical Informatics chapter of this year's Yearbook, Dr Kulikowski provides historical insight into the scientific, technological, and practical clinical accomplishments of Donald Lindberg. How Dr Lindberg opened free access and worldwide public dissemination of all the NLM's biomedical literature and databases is presented as an example of ethical scientific openness checking the four pillars autonomy, justice, beneficence, and non-maleficence.

\section{IMIA President's Statement}

Dr Sabine Koch provided her inaugural IMIA President's Statement. Beyond some very thoughtful reflections about the rapidly evolving biomedical and health informatics field and the continuously arising new challenges that have to be faced, she offered some important information about IMIA. One piece of importance is the formal statement submitted by the IMIA's academy to the World Health Organization (WHO) in response to the current COVID-19 global pandemic. The statement emphasizes the use of health informatics methodology and information and communication technology in combating the current COVID-19 pandemic and future outbreaks. Other IMIA's initiatives are described including the preparation of MEDINFO 2021.

\section{IAHSI Papers}

The 2020 edition of the IMIA Yearbook includes two papers from the International Academy of Health Sciences Informatics (IAHSI). The first one authored by the members of the Academy's first Board including elected and ex officio members provides a summary of the major Academy activities in 2018 and 2019 and an outline of the actions planned for 2020, showing that the Academy is completely established and that progress is now on track. The second paper is an edited version of the Academy 'Strategy and Focus areas' document reflecting major outcomes of intensive discussions that occurred during 2019 and presented at the Academy's 3rd Plenary during MEDINFO 2019. Regardless the 'living document' nature of the 'Strategy and Focus areas' document, Academy Fellows decided that this current first version describing IAHSI vision, mission, principles, values, and strategic directions would be used as a base for decisions on future activities.

\section{Changes in the Yearbook Editorial Committee}

As every year, some changes would occur in the Yearbook editorial team. In 2020, the two co-editors of the Decision Support (DS) section were Vassilis Koutkias, researcher in Biomedical Informatics at CERTH/INAB (Thessaloniki, Greece) and Catherine Duclos, Professor in Biomedical Informatics at Sorbonne Paris Nord University (Paris, France). We are really sad and it is painful to remind Yearbook readers that Vassilis passed away in December 2019 leaving a huge void in our hearts and a deep sorrow for many members of the Yearbook Editorial Committee. An obituary in his memory is included in this year's Yearbook. We want to thank Jacques Bouaud, researcher in Biomedical Informatics at the LIMICS (Paris, France), who has been serving as DS section co-editor since 2012. Jacques has been a true pillar of the Editorial Committee, always available for any section editor, especially to help in the implementation of the Yearbook method to conduct the literature search for the selection of best papers. Despite he decided to leave the Editorial Committee in 2019, he accepted to work with Catherine Duclos to replace Vassilis and finish the 2020 work on the DS section.

Our thanks go also to the two editors of the Bioinformatics and Translational Informatics section, Malika Smail-Tabbone, Associate Professor of Computer Science at Lorraine University (Nancy, France), and Bastien Rance, Associate Professor of Biomedical Informatics at Paris University (Paris, France) that would leave the Yearbook Editorial Committee in 2021. We also want to thank the co-editors of the Public Health and Epidemiology Informatics (PHEI) section, Rodolphe Thiébaut, Professor of Public Health and Biostatistics at the University of Bordeaux (France) and Sébastien Cossin, Assistant in Public Health Informatics at the University of Bordeaux (France) that leave the Yearbook Editorial Committee. In 2021, the special topic of the Yearbook is "Managing pandemics with Health Informatics: successes and challenges", thus there will not be a PHEI section on top of the special section. 
Finally, after four years as a chief editor (2012-2016), and four years as IMIA VP Services (2016 - 2020), Brigitte Séroussi is leaving the Yearbook Editorial Committee on November 2020. A new IMIA VP for Services will be elected at the next IMIA General Assembly meeting that will take place in Hamamatsu (浜松) (Japan) during the APAMI 2020,1 $11^{\text {th }}$ Biennal Conference of the Asia-Pacific Association for Medical Informatics.

\section{References}

1. Friedman CP, Rubin JC, SullivanKJ. Toward an Information Infrastructure for Global Health Improvement. Yearb Med Inform 2017 Aug;26(1):16-23.

2. https://www.statnews.com/2020/07/15/artificial-intelligence-patient-consent-hospitals/ (last access on July 20th)

3. Na L, Yang C, Lo CC, Zhao F, Fukuoka Y, Aswani A. Feasibility of Reidentifying Individuals in Large National Physical Activity Data Sets From Which Protected Health Information Has Been Removed With Use of Machine Learning. JAMA Netw Open 2018 Dec 7;1(8):e186040.

4. Lunshof JE, Chadwick R, Vorhaus DB. From genetic privacy to open consent. Nat Rev Genet. 2008 May;9(5): 406-11.

5. Kitchin, R. Big Data, new epistemologies and paradigm shifts. Big Data \& Society 2014;1(1): 1-12.

6. https://ec.europa.eu/digital-single-market/en/ news/ethics-guidelines-trustworthy-ai (last access on July 20th).

7. Kaplan B. How Should Health Data Be Used? Privacy, Secondary Use, and Big Data Sales. Camb Q Healthc Ethics 2016 Apr;25(2):312-29.

8. Campos-Castillo C, Anthony DL. The double-edged sword of electronic health records: implications for patient disclosure. J Am Med Inform Assoc 2015 Apr;22(e1):e130-40. 